\title{
Using magnetic resonance microscopy to study the growth dynamics of a glioma spheroid in collagen I: A case study Shuning Huang1,2, David Vader ${ }^{3}$, Zhihui Wang1, Anat Stemmer- Rachamimov ${ }^{4}$, David A Weitz ${ }^{3}$, Guangping Dai ${ }^{1}$, Bruce R Rosen ${ }^{1,2}$ and Thomas S Deisboeck*1,2,3
}

\author{
Address: ${ }^{1}$ Harvard-MIT (HST) Athinoula A. Martinos Center for Biomedical Imaging, Massachusetts General Hospital, Charlestown, MA 02129, \\ USA, ${ }^{2}$ Harvard-MIT (HST) Massachusetts Institute of Technology, Cambridge, MA 02139, USA, ${ }^{3}$ chool of Engineering and Applied Sciences \\ (SEAS), Harvard University, Cambridge, MA 02138, USA and ${ }^{4}$ Department of Pathology (Neuropathology), Massachusetts General Hospital, \\ Boston, MA 02114, USA \\ Email: Shuning Huang - shuning@mit.edu; David Vader - vader@fas.harvard.edu; Zhihui Wang - billwang@nmr.mgh.harvard.edu; \\ Anat Stemmer-Rachamimov - astemmerrachamimov@partners.org; David A Weitz - weitz@ deas.harvard.edu; \\ Guangping Dai - dai@nmr.mgh.harvard.edu; Bruce R Rosen - bruce@nmr.mgh.harvard.edu; \\ Thomas S Deisboeck* - deisboec@helix.mgh.harvard.edu \\ * Corresponding author
}

Published: 29 January 2008

BMC Medical Imaging 2008, 8:3 doi:10.1 186/147/-2342-8-3

This article is available from: http://www.biomedcentral.com/I47/ -2342/8/3

(C) 2008 Huang et al; licensee BioMed Central Ltd.

This is an Open Access article distributed under the terms of the Creative Commons Attribution License (http://creativecommons.org/licenses/by/2.0), which permits unrestricted use, distribution, and reproduction in any medium, provided the original work is properly cited.
Received: 5 June 2007

Accepted: 29 January 2008

\begin{abstract}
Background: Highly malignant gliomas are characterized by rapid growth, extensive local tissue infiltration and the resulting overall dismal clinical outcome. Gaining any additional insights into the complex interaction between this aggressive brain tumor and its microenvironment is therefore critical. Currently, the standard imaging modalities to investigate the crucial interface between tumor growth and invasion in vitro are light and confocal laser scanning microscopy. While immensely useful in cell culture, integrating these modalities with this cancer's clinical imaging method of choice, i.e. MRI, is a non-trivial endeavour. However, this integration is necessary, should advanced computational modeling be able to utilize these in vitro data to eventually predict growth behaviour in vivo. We therefore argue that employing the same imaging modality for both the experimental setting and the clinical situation it represents should have significant value from a data integration perspective. In this case study, we have investigated the feasibility of using a specific form of MRI, i.e. magnetic resonance microscopy or MRM, to study the expansion dynamics of a multicellular tumor spheroid in a collagen type I gel.
\end{abstract}

Methods: An U87mEGFR human giloblastoma multicellular spheroid (MTS) containing approximately $4 \cdot 10^{3}$ cells was generated and pipetted into a collagen I gel. The sample was then imaged using a $T_{2}$-weighted $3 \mathrm{D}$ spoiled gradient echo pulse sequence on a I4T MRI scanner over a period of 12 hours with a temporal resolution of 3 hours at room temperature. Standard histopathology was performed on the MRM sample, as well as on control samples.

Results: We were able to acquire three-dimensional MR images with a spatial resolution of $24 \times$ $24 \times 24 \mu \mathrm{m}^{3}$. Our MRM data successfully documented the volumetric growth dynamics of an MTS in a collagen I gel over the I2-hour period. The histopathology results confirmed cell viability in the MRM sample, yet displayed distinct patterns of cell proliferation and invasion as compared to control. 
Conclusion: In this study, we demonstrate that a specific form of MRI, i.e. magnetic resonance microscopy or MRM, can be used to study the dynamic growth of a multicellular tumor spheroid (MTS) with a single cell scale spatial resolution that approaches the level of light microscopy. We argue that MRM can be employed as a complementary non-invasive tool to characterize microscopic MTS expansion, and thus, together with integrative computational modeling, may allow bridging of the experimental and clinical scales more readily.

\section{Background}

High-grade malignant gliomas are characterized by rapid volumetric growth and extensive local tissue infiltration. Despite all efforts to improve diagnostics and therapy, the outcome remains dismal with a five-year survival rate below 3.3\% in the main age group of 45-years and older [1]. Since the surrounding tissue is thought to impact, perhaps even guide the tumor's invasive patterns, much weight is currently being put on better understanding the dynamic interaction of an expanding brain tumor with its microenvironment. As such, following an interdisciplinary approach, we have previously employed an in vitro glioma multicellular tumor spheroid (MTS) model to investigate the spatial and temporal dynamics of MTS expansion within extracellular matrix environments [2-4], and started to model them in silico [5-7]. In these studies, various aspects of the interaction between a growing spheroid system and its environment were studied and mathematically modeled, including extracellular matrix concentration, mechanical forces, and invasion directionality.

In the 1980s, several groups independently acquired microscopic images of different biological samples using magnetic resonance spectroscopy [8-10]. These early studies demonstrated the feasibility of magnetic resonance microscopic imaging with its unique sensitivity to tissue water environment. Since then, with continued advances in magnetic resonance imaging (MRI) techniques (incl. stronger gradient coils, improved RF coil design, and more powerful computers), the imaging resolution has steadily increased. We are now able to acquire threedimensional (3D) images at a high signal to noise ratio with a resolution approaching the single cell level. This allows us to employ MRI, the imaging modality of choice in vivo, to study these microscopic tumor models also in vitro.

We have previously reported that magnetic resonance microscopy (MRM) enables us to distinguish an MTS of roughly $250 \mu \mathrm{m}$ in diameter in a collagen I gel and provides us with a true $3 \mathrm{D}$ view of the MTS within the matrix [11]. However, the time required to obtain high-resolution 3D images was considerably long, typically 10 hours. To dynamically monitor MTS growth, we would have to increase the temporal resolution while maintaining a comparable signal to noise ratio (SNR). For this very purpose, we applied Gd-DTPA, the contrast agent widely used in clinics, to the collagen I matrix, achieving not only a significantly shortened $\mathrm{MR}$ imaging time down to 3 hours, but also increasing the SNR from 20 to over 40 . Therefore, we are able to dynamically monitor both global and local changes of the MTS over a 12-hour period at a temporal resolution of 3 hours and an isotropic spatial resolution of $24 \mu \mathrm{m}$. Using conventional immunohistochemistry techniques we were able to confirm cell viability in the MRM sample post imaging, as well as to find patterns of cell proliferation and invasion that seem distinctively different from the controls and thus warrant further analyses.

In summary, we argue that MRM enables us to examine MTS growth dynamically with a true $3 \mathrm{D}$ view. The nature of MRM data allows us to segment the spheroid's contour out of its microenvironment, reconstruct and visualize its surface, and subsequently analyze the MTS' distinct 3D dynamics from any selected angle. Tracking tumor expansion non-invasively down to the 'single-cell' scale by using a clinically relevant imaging modality should facilitate data integration, and, in combination with in silico modeling, will yield valuable insights into the critical interaction between the tumor and its microenvironment.

\section{Methods \\ Multicellular tumor spheroid (MTS) and extracellular matrix \\ Cell culture}

The U87mEGFR [11-13] cell line is cultured in $10 \mathrm{~mL}$ of high-glucose Dulbecco's Modified Essential Medium (DMEM; Invitrogen, Carlsbad, CA), supplemented with 1\% Penicillin-Streptomycin (PS; Invitrogen), 10\% Fetal Bovine Serum (FBS; JRH Biosciences, Lenexa, KS), $0.5 \mathrm{mg} /$ $\mathrm{mL}$ Geneticin (Invitrogen) and $20 \mathrm{mM}$ hepes buffer (Invitrogen) in $10 \mathrm{~cm}$ diameter Petri dishes (Corning, Corning, NY). Once cells are confluent, they are twice rinsed with Phosphate Buffered Saline (Invitrogen) and detach after the addition of $1 \mathrm{~mL}$ Trypsin-EDTA (Invitrogen). After 5-10 min, $9 \mathrm{~mL}$ of fresh cell media is added to neutralize the Trypsin. The cell solution is then transferred into a $15 \mathrm{~mL}$ centrifuge tube and centrifuged for $5 \mathrm{~min}$ at $1200 \mathrm{RPM}\left(\sim 2 \cdot 10^{3} \mathrm{~m} / \mathrm{s}^{2}\right)$. After aspirating the superna- 
tant, the cells are resuspended in fresh media at a concentration of $2 \cdot 10^{5} / \mathrm{mL}$.

\section{Multicellular spheroids}

Spheroids are then generated using the hanging droplet method [14], with a drop size of $20 \mu \mathrm{L}$, which seeds $\sim 4 \cdot 10^{3}$ cells in each spheroid to yield a diameter of approximately $400 \mu \mathrm{m}$. Briefly, multiple droplets of $20 \mu \mathrm{L}$ of cell solution are pipetted onto the inside of a $100 \mathrm{~mm}$ diameter Petri dish cover. After placing the cover back on a culture medium-filled dish, the dish itself is placed in the incubator $\left(5 \% \mathrm{CO}_{2}, 37^{\circ} \mathrm{C}\right)$. Surface tension maintains droplet integrity, while gravity pulls cells together at the bottom of each droplet. Cells are left to form spheroids in the incubator for 3 days before they are collected.

\section{Extracellular matrix}

The extracellular matrix (ECM) model is a $1.5 \mathrm{mg} / \mathrm{mL}$ bovine collagen type I (Inamed Biomaterials, Fremont, CA) matrix supplemented with 10\% FBS, 10\% 10X Minimum Essential Medium (MEM; Invitrogen), 1\% PS, 50 $\mathrm{mM}$ sodium bicarbonate $\left(\mathrm{NaHCO}_{3}\right.$; Sigma, St. Louis, $\mathrm{MO}$ ) buffer. For image enhancement, $10 \mathrm{mM}$ Gd-DTPA (Magnevist; Berlex, Wayne, NJ, U.S.A.) is added to the collagen solution. To induce polymerization, a few $\mu \mathrm{L}$ of $1 \mathrm{M}$ sodium hydroxide ( $\mathrm{NaOH}$; Sigma) are added until a neutral $\mathrm{pH}$ is reached. The choice of $1.5 \mathrm{mg} / \mathrm{mL}$ concentration used here is based on work by Kaufman et al. [2] and represents a reasonable compromise between achieving a sufficiently high viscoelastic modulus while avoiding blocking cell motility altogether.

\section{Sample preparation}

For each sample, we pipette $280 \mu \mathrm{Ls}$ of collagen solution into a $\mu$ PCR tube (VWR, West Chester, PA). After 15-30 min in the incubator to allow the gel to initiate polymerization, a spheroid is pipetted into this collagen gel, which is then placed back in the incubator for several hours to allow cells to attach properly to the ECM before imaging. To assess the impact of MRM on cell viability, we compared the histology of the MR-imaged specimen with a control group that was treated identically but for one difference: instead of being imaged overnight, it was kept in the incubator. As soon as the experiment was halted, both the imaged specimen and the control samples were fixed, sectioned and stained.

\section{Magnetic resonance microscopy}

We first measured Gd-DTPA (Magnevist) relaxivity in water at 14T (Magnex, $89 \mathrm{~mm}$ vertical bore, gradient strength 100 gauss $/ \mathrm{cm}$, Bruker Biospin System) using the conventional inversion recovery spin echo sequence at three different concentrations $(0.075,2.5,5 \mathrm{mM})$. The Gd-DTPA concentration $(10 \mathrm{mM})$ used here was then determined based on its dosage $(0.2-0.5 \mathrm{mmol} / \mathrm{kg})$ in MRI mouse model studies $[15,16]$ and its relaxivity at $14 \mathrm{~T}$.

The MTS in collagen with $10 \mathrm{mM}$ Gd-DTPA was imaged using MRM at 14T. Specifically, we first used a modified FLASH pulse sequence to acquire multi-slice multi-echo $(\mathrm{TR} / \mathrm{TE}=400 / 381318 \mathrm{~ms} ; \mathrm{FOV}=0.95 \mathrm{~cm}, 256 \times 256$; slice thickness $=400 \mu \mathrm{m} ; \alpha=30$ ) images with an in-plane resolution of $37 \times 37 \mu \mathrm{m}^{2}$ to localize the MTS in the collagen I gel. A quick $\mathrm{T}_{1}$ measurement was then performed using a modified IR_RARE sequence (TR/TE: 1500/7.86 ms; rare factor: 2 ; matrix size: $128 \times 128$; 10 slices with 0.4 $\mathrm{mm}$ slice thickness; FOV: $0.6 \mathrm{~cm}$; TI: 5.62155 .620 $105.620205 .620505 .6201005 .6202005 .620 \mathrm{~ms})$. A three-dimension spoiled gradient echo (FLASH) sequence $(\mathrm{TR} / \mathrm{TE}=20 / 5.5 \mathrm{~ms} ; \mathrm{FOV}=1.2 \times 0.6 \times 0.6$; matrix size: $512 \times 256 \times 256$; signal was optimized at the Ernst angle) was then used to acquire high-resolution $(24 \mu \mathrm{m}$ isotropic) microscopic images every three hours for a total of 12 hours. After imaging, the sample was fixed for histopathological analysis as described below. MR images were displayed employing an Image software package [17] and then segmented using the 3D Slicer software package [18]. $\mathrm{T}_{1}$ of the sample and Gd-DTPA relaxivity were fitted using Matlab (Mathwork, Inc., Natick, MA). After the MTS was manually segmented for each time point, its corresponding volume and surface area were calculated.

\section{Histology}

The spheroids were harvested and processed as described before [4], fixed in 10\% formalin and embedded in paraffin. Blocks were serially sectioned in $7 \mu \mathrm{m}$ thick sections and stained with hematoxylin and eosin (H\&E). In order to assess the proliferative activity, immunohistochemistry was performed by using cell cycle-unspecific MIB1 antibody (which detects the nuclear Ki-67 antibody so that quiescent cells (G0) remain unstained) (DAKO, M7240, USA; 1:50 dilution). The so called MIB-1 labeling index was then calculated as the fraction of MIB-1 positive cells from the total number of cells. In addition, images of the test specimen and control were printed. The number of invading cells was counted and the distance of each cell was calculated from a circle drawn at the circumference of the spheroid. The distances were measured by drawing the shortest straight line from the circle around the spheroid to the invading cells. The measurements are in $\mathrm{cm}$, but do not represent true distance of invasion (due to magnification of the image printed), but rather represent an [arbitrary unit] by which distances of the cells from the two MTS, i.e. 14T specimen versus control, can be compared. 


\section{Results}

Magnetic resonance microscopy of MTS in collagen I gel Prior to imaging the MTS, we characterized the $\mathrm{r}_{1}$ relaxivity (longitudinal relaxivity) of Gd-DTPA at $14 \mathrm{~T}$ and the SNR improvement after adding $10 \mathrm{mM}$ Gd-DTPA into the collagen I gel that did not contain any tumor cells. Table 1 shows that with $10 \mathrm{mM}$ Gd-DTPA, we were able to reduce the $\mathrm{T}_{1}$ relaxation time of the collagen gel from around $3000 \mathrm{~ms}$ to about $24 \mathrm{~ms}$. The imaging time was reduced from 10 hours to 3 hours with a two-fold increase of SNR (from 20 to over 40 ).

The original MRM images of the MTS specimen are shown in Figure 1. The time series clearly documents the growth of the spheroid from different angles and at different planes. Even with the 2D view, MRM showed different patterns of cellular growth. Images on the left in Figure 1 show cells growing attached to the main body (red circle); while those on the right show some cells seemingly moving away from the main spheroid. It becomes apparent that with reduced imaging time and increased SNR, MRM can indeed monitor the spatio-temporal expansion of an MTS system.

\section{Segmentation and 3D visualization of MTS}

The 3D reconstruction shows rather heterogeneous expansion patterns with rough surface growth areas throughout over the course of the observation period (Figure 2(A)). Intriguingly, once started (orange droplets at the MTS' proximal apex at time point ' 3 hours') glioma cell expansion into the gel seems to give rise to an 'imprinting' process which confirms the 'trailblazer' concept that has been described previously in $[2,4]$. Finally, corresponding to the MTS' preserved structural viability (compare with Fig. 3) Figure 2(B) also confirms its functionality in that both MTS volume and surface area increase throughout the observation period.

\section{Histopathology}

To semi-quantitatively evaluate the viability of the MTS that has been imaged over 12 hours with 14T MRM, standard immunohistochemistry was performed and the histopathology results were compared with the control (as described above). As documented in Figure 3, the imaged MTS not only remains viable overall but, compared to the control tumors the $14 \mathrm{~T}$ specimen even contained a sub- stantially larger proliferative fraction (see Table 2). More specifically, the MIB-1 labeling index for the control is $12.5 \%$ (30 positive cells out of 239 counted) as compared to the MIB-1 labeling index for the specimen which is with $21.1 \%$ (50 positive cells out of 236 cells counted) almost doubled. Furthermore, reviewing comparable sections, in the $14 \mathrm{~T}$ specimen only 5 cells could be found to invade the gel; conversely, a [total] of 32 cells invaded the collagen matrix in the control experiment. While the min-max values expectedly (given the difference in $n$ ) showed considerably more heterogeneity in the control group, intriguingly, the overall mean invasion distance was with 2.10 versus 2.11 almost identical between $14 \mathrm{~T}$ specimen and control. We note that a $2^{\text {nd }}$ control experiment confirmed the results.

\section{Discussion}

Our data provide clear evidence that magnetic resonance microscopy can be used to study the dynamic growth of multi-cellular tumor spheroid embedded in a collagen I matrix, at a resolution close to the single cell level (Figure 1). Adding $10 \mathrm{mM}$ Gd- DTPA to the gel dramatically reduced the image acquisition time without any detrimental impact on cell viability (Figure 3). Intriguingly, the glioma cells showed distinct spatio-temporal expansion patterns into the gel with spotty surface expansion across the spheroid and the notion of a 'trailblazer' mechanism that guides cell motility in that single cells follow each other along preformed pathways (Figure 2A). The histological results showed surprisingly not only more proliferative activity yet concomitantly also less cells pursuing invasion in the MRM specimen than in the control. Intriguingly, the mean invasive distance was virtually identical which supports the notion that the phenotype itself remained unaffected. This preliminary data support the notion of a "dichotomy" between proliferation and migration, which was experimentally shown by Giese et al. [19] and modeled with a molecular switching mechanism by Athale et al [20] and Zhang et al [21]. The impact of high field MR (14 Tesla in our study) on cancer cells is somewhat controversial. For instance, Santini et al [22] showed that a sinusoidal $50 \mathrm{~Hz}$ magnetic field of $1 \mathrm{mT}$ significantly increased spheroid invasive properties, but had no damage on growth. Short et al. [23] showed that for the two cell lines exposed to a $4.7 \mathrm{~T}$ field up to 72 hours, there was no change in cell growth rate. On the contrary,

Table I: MRI properties of Gd-DTPA and collagen I gel.

\begin{tabular}{|c|c|c|c|}
\hline & Gd-DTPA & Collagen I Gel without $10 \mathrm{mM} \mathrm{Gd}$ & Collagen I Gel with $10 \mathrm{mM} \mathrm{Gd}$ \\
\hline rl (I mM-1 sec-1) & $3.88 \pm 0.12$ & & \\
\hline $\mathbf{R}_{\mathbf{l}}\left(\mathrm{sec}^{-1}\right)$ & & 0.33 & 42.32 \\
\hline SNR & & 16 & 42 \\
\hline
\end{tabular}

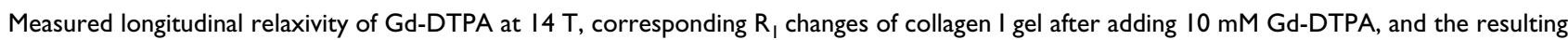
SNR improvement. 

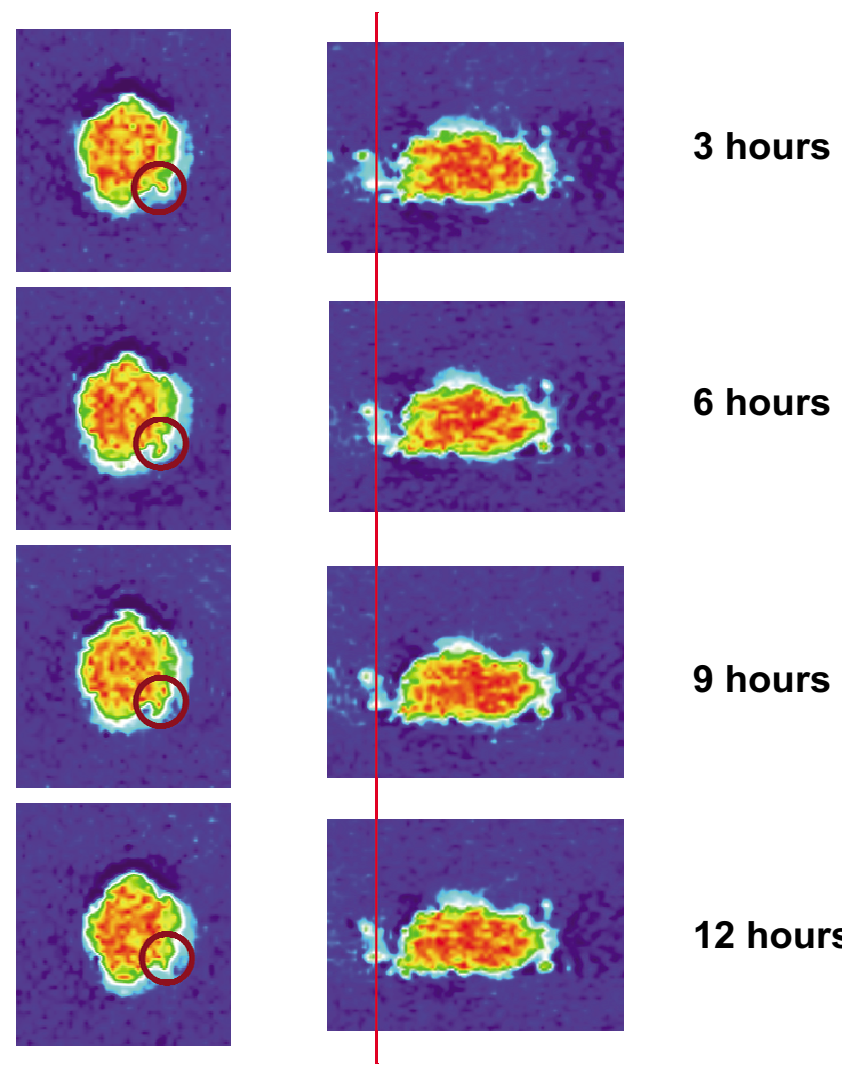

6 hours

9 hours

12 hours

\section{Figure I}

MRM images that show the MTS growth over time from different angles. The images to the left depict the axial view, whereas those to the right show an arbitrary angle. These $2 \mathrm{D}$ images confirm the surface heterogeneity that emerges already $12 \mathrm{hrs}$ post placement of the MTS into the gel, and depict a small group of invasive cells (images on the right) that can also be seen in Figures 2(A) and 3. On the other hand, images on the left demonstrate that certain parts of the solid spheroid actually grew into the gel (red circle).

Raylamn et al [24] demonstrated that human cancer cells exposed to $11.7 \mathrm{~T}$ for 64 hours had a slower growth rate. In our study, the spheroid also experienced a high frequency small magnetic field ( $B_{1}$ field, RF pulse) in addition to the $14 \mathrm{~T}$ main magnetic field. Although the results from a single case study must be interpreted with proper caution, the increase in proliferation and concomitant reduction in the number of invasive cells observed in our study may indicate that different exposure time and magnetic field strength can impact tumor expansion patterns. Hence, our results warrant a more extended study to investigate in detail the impact of MRM on normal and cancer cell viability and phenotypes.

Kaufman et al [2] showed different patterns of MTS growth and mobility in collagen I matrices of varying con- centrations. They observed a more effective invasion in high concentration $(1.5$ and $2.0 \mathrm{mg} / \mathrm{mL})$ gels at early time. The choice of $1.5 \mathrm{mg} / \mathrm{mL}$ concentration used here was a compromise between having a high modulus to maintain sample integrity and allowing cells to move around quickly, and low optical density to allow us to locate the spheroid easily by eye and enable, perhaps, comparisons with optical imaging in the future. Previously, Brandl et al [25] used an 11.7T spectrometer to study multicellular tumor spheroids that were generated from human malignant MV3 melanoma. In their study, the spheroid was allowed to grow for seven to fourteen days in agar to a diameter of $400-1000 \mu \mathrm{m}$ with a starting dense population of $5 \cdot 10^{6}$ cells. This may lead to central necrosis due to limited nutrition and oxygen in the core region. They indeed observed a necrotic core in their sample. The fact that the authors did not observe any morphologic change over the four to five-hour imaging time is less surprising since their agar environment is far more rigid than the collagen I gel used in our study here.

The advantage of using MRM in this context lies in its noninvasiveness, and its true three-dimensional full-scale (global and local) view of MTS' dynamic growth. It is very difficult, if not impossible to acquire whole three-dimension images of MTS in collagen I gel using most modern optical imaging methods. Confocal microscopy allows three-dimensional imaging; however, traditional single photon reflectance or fluorescence methods are limited by the working distance of objectives, typically a few hundred microns. Two-photon confocal microscopy and second harmonic generation can go beyond this limit, but still remain within $1 \mathrm{~mm}$ of the surface [26-28]. Conventional light microscopy can surpass this limitation as well, but this is accompanied by a loss in axial resolution. Moreover, a general limitation of light microscopy is that it is constrained by the opacity of tissues. To overcome the loss of signal due to increasing depth, one has two choices - with clear drawbacks: i) increasing light power may decrease cell viability very quickly, together with the photobleaching seen in typical fluorescence imaging; ii) fixing and staining the cells prior to imaging usually yields a better signal-to-noise ratio than live cells, but the mere fact of fixing the system is an obvious loss in evaluating the time evolution of a living sample. That is not to say of course that optical methods are incapable of generating useful and quantitative information. Interesting studies by e.g. Nygaard et al [29] and recent work by Kaufman et al [2] yield useful information about MTS growth, though with the limitations described above.

With the presently achieved MRM resolution already, we observe "spotty" volumetric changes. Future studies of MTS growth using MRM will reveal even more detailed structural changes in a true $3 \mathrm{D}$ sense as the image resolu- 

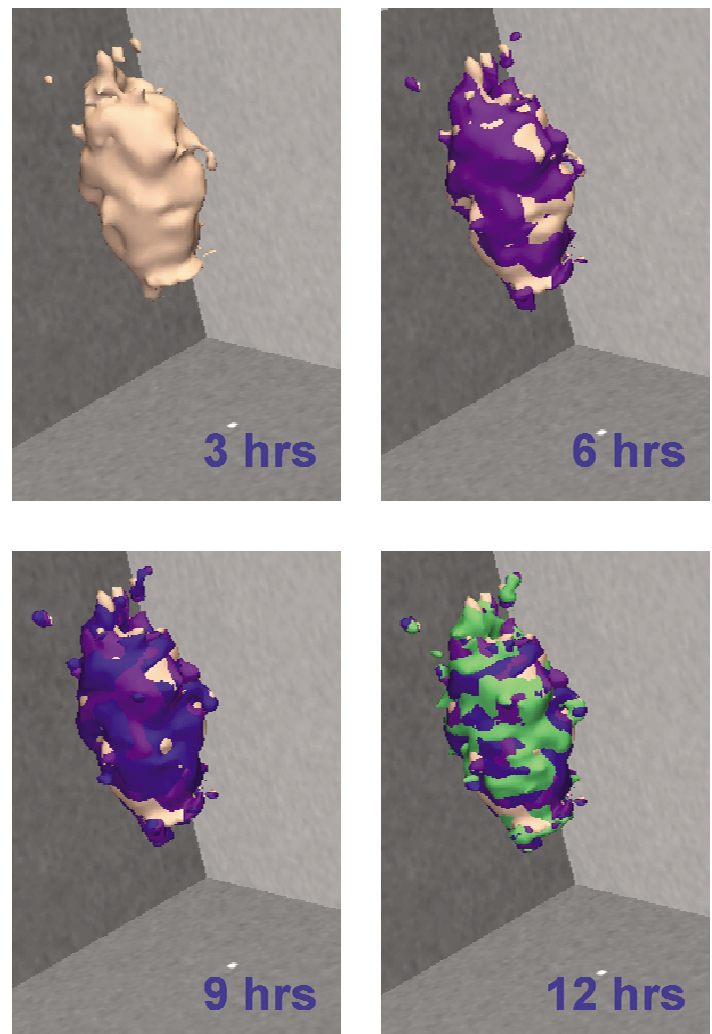

A

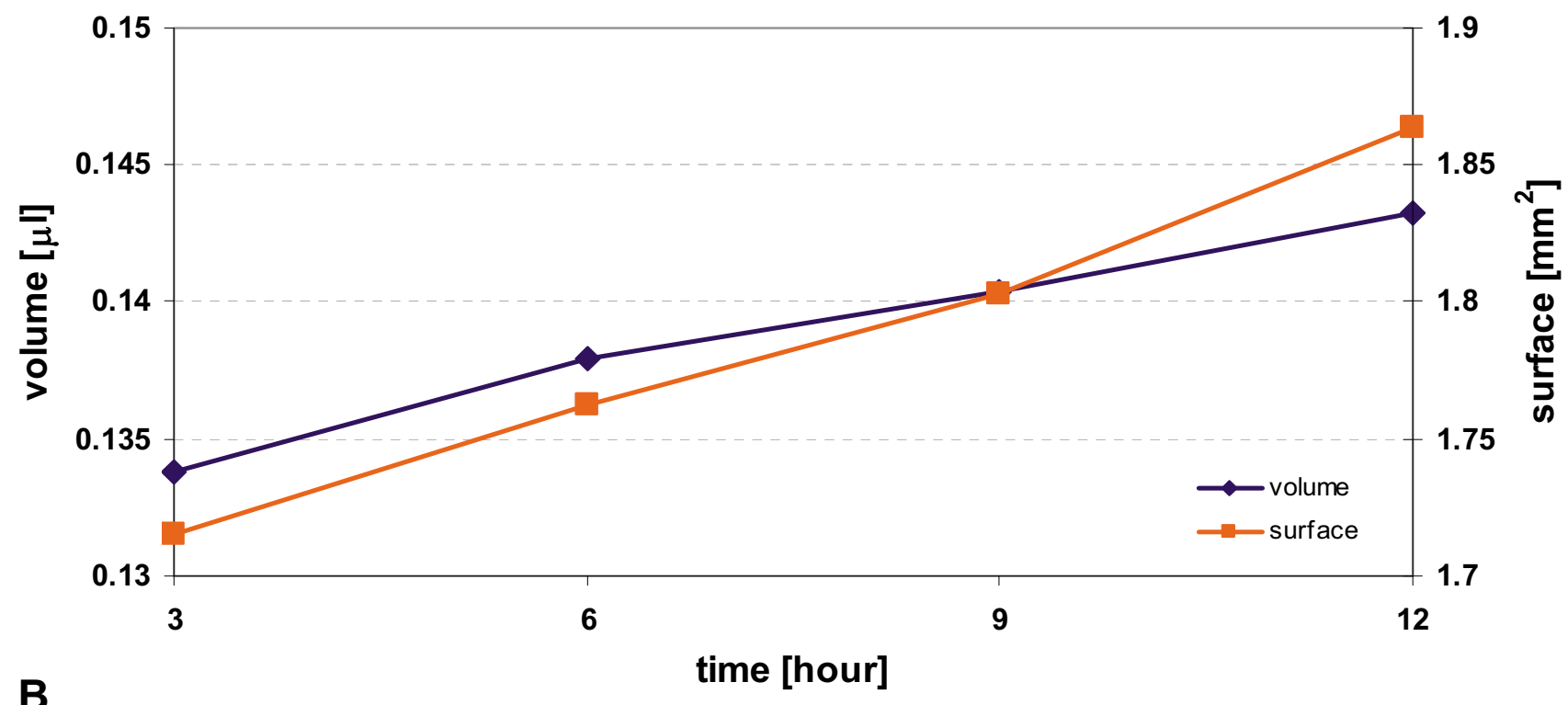

Figure 2

Volumetric rendering and growth dynamics of the MTS in the collagen I matrix at four consecutive time points. (A) The segmented MTS is reconstructed in 3D and the color-coding indicates the growth increase at each time point. The MTS appears to grow anistropic, which may indicate regional heterogeneities in composition of either MTS or microenvironment, or both, and/or point towards a heterogeneous local interaction between cells and gel. (B) MTS volume and surface area are calculated and plotted over time. 


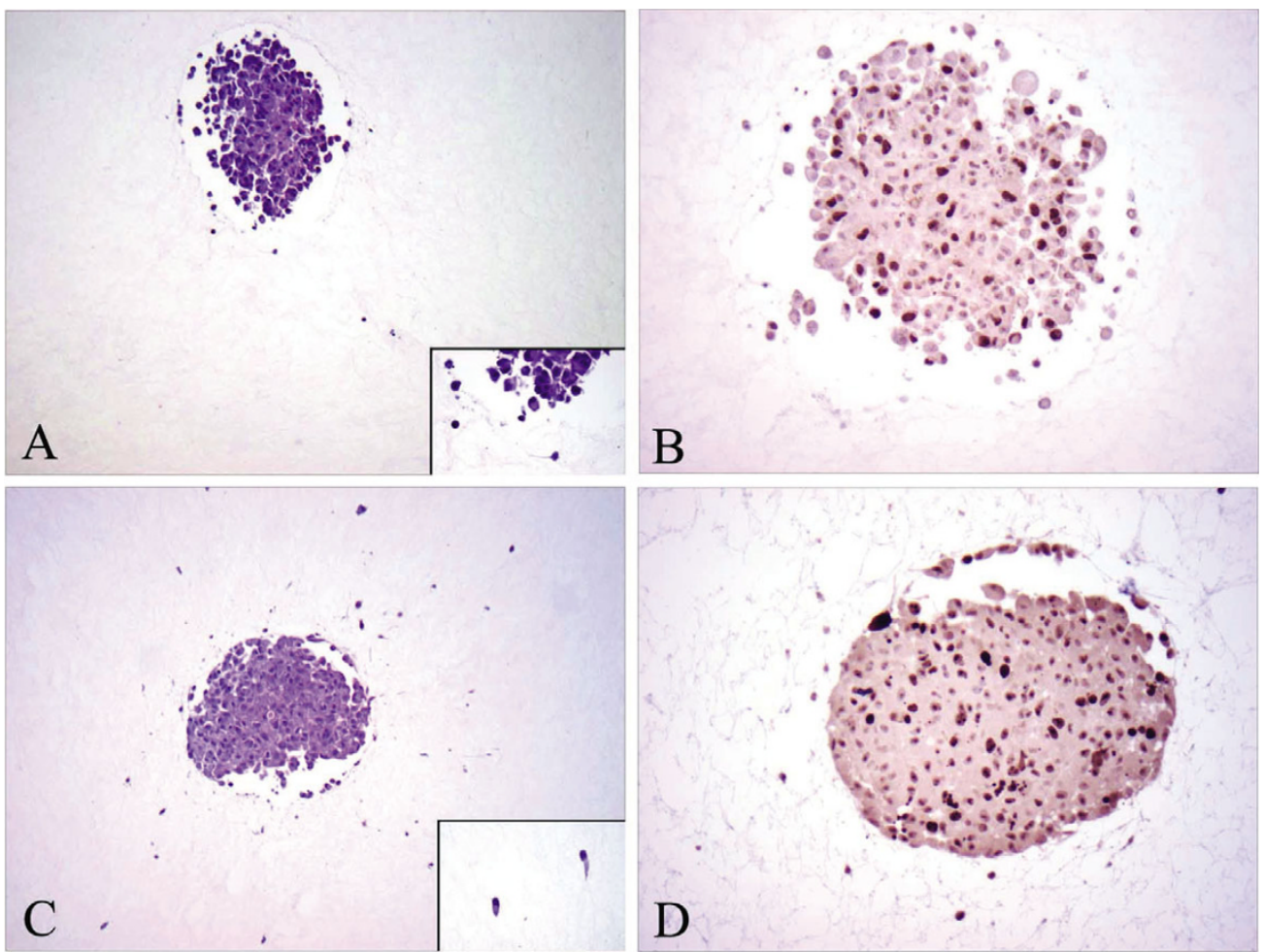

Figure 3

Histopathology findings, comparing I4T MTS with control. (A) H\&E staining of I4T specimen (original magnification $\times 100$ ) showing only a small number of cells infiltrating the gel. Insert shows infiltrating cells in higher magnification (original magnification $\times 400)$. (B) MIB-I immunostaining of I4T specimen (original magnification $\times 200$ ), highlighting cells that are not in G0 as dark brown nuclei. (C) H\&E staining of control (original magnification $\times 100$ ) showing a large number of infiltrating cells in adjacent gel. Insert shows the infiltrating cells in higher magnification (original magnification $\times 400$ ). (D) MIB-I immunostaining of control (original magnification $\times 200$ ), highlighting cells that are not in $\mathrm{G0}$ as dark brown nuclei.

tion further improves. MRM is beginning to bridge the imaging scale that, until recently, has been accessible to optical techniques only, with the clinical MRI data level which is still plagued by a rather poor spatial and temporal resolution. Such three-dimensional volumetric studies of the morphologic growth of MTS using MRM will provide complementary information about the growing tumor and the dynamic interaction with its microenvironment. With the help of integrative in silico modeling insights gained with MRM in vitro can help us understand better the complex cancer growth patterns seen in patients.

\section{Conclusion}

We have shown that magnetic resonance microscopy can be used to study dynamic tumor growth in vitro with a resolution that approaches that of light microscopy. Based on this microscopic MRI pilot data, we argue that one may be able to use this clinically relevant imaging modality also in vitro, particularly once combined with molecular imaging techniques. Being able to rely on one imaging modality only, or even primarily, would greatly enhance our ability to successfully integrate in vitro with in vivo data, using innovative in silico models that bridge between these scales.

Table 2: Histopathology.

\begin{tabular}{|c|c|c|c|c|c|c|}
\hline \multirow[b]{2}{*}{ I4T } & \multicolumn{2}{|c|}{ Proliferation } & \multirow[b]{2}{*}{$\mathrm{n}: \mathbf{5}$} & \multirow[b]{2}{*}{ Mean: 2.10 } & \multirow{2}{*}{$\begin{array}{l}\text { Invasion } \\
\text { SD: } 2.07\end{array}$} & \multirow[b]{2}{*}{ Min-Max: $0.50-4.70$} \\
\hline & $50 \mathrm{pos} / 236$ & $21.1 \%$ & & & & \\
\hline Control & $30 \mathrm{pos} / 239$ & $12.5 \%$ & $\mathrm{n}: 32$ & Mean: 2.I I & SD: 1.60 & Min-Max: 0.30-6.50 \\
\hline
\end{tabular}

To document proliferative activity, we report here the percentage of MIB-I positive cells for the I4T specimen versus control. The invasion data result from measuring the straight-line distance between an invasive cell and a circle drawn around the spheroid. The measurements are done on a print of a $20 \times$ magnification, and represent [arbitrary units]. (See text for more details). 


\section{Competing interests}

The author(s) declare that they have no competing interests.

\section{Authors' contributions}

SH carried out the MRM studies and participated in the image analysis. GD was responsible for the MRI pulse sequence development. DV was responsible for sample preparation and cell culture, whereas ZW carried out the $3 \mathrm{D}$ image reconstruction and calculated the growth dynamics. ASR performed and interpreted the histopathological and immunohistochemical analyses. BRR and DAW helped direct the MR imaging and materials science aspects involved. TSD conceived the study, led its overall design and coordination, and helped to draft the manuscript. All authors read and approved the final manuscript.

\section{Acknowledgements}

This work has been supported in part by NIH grants CA 085 I39, CA I I 3004 (The Center for the Development of a Virtual Tumor, CViT [30]), MIND and NCRR (P4IRR I4075) and by the Harvard-MIT (HST) Athinoula A. Martinos Center for Biomedical Imaging and the Department of Radiology at Massachusetts General Hospital. We thank Ms. Candice A. Romany (Department of Pathology, Massachusetts General Hospital) for processing the histopathology specimen.

\section{References}

I. CBTRUS: Statistical report: Primary brain tumors in the United States statistical report, 1 998-2002 (Years Data Collected). The Central Brain Tumor Registry of the United States (CBTRUS); 2005-2006

2. Kaufman LJ, Brangwynne CP, Kasza KE, Filippidi E, Gordon VD, Deis boeck TS, Weitz DA: Glioma expansion in collagen I matrices: analyzing collagen concentration-dependent growth and motility patterns. Biophys J 2005, 89(I):635-650.

3. Gordon VD, Valentine MT, Gardel ML, Andor-Ardo D, Dennison S, Bogdanov AA, Weitz DA, Deisboeck TS: Measuring the mechanical stress induced by an expanding multicellular tumor system: a case study. Experimental Cell Research 2003, 289(I):58-66.

4. Deisboeck TS, Berens ME, Kansal AR, Torquato S, Stemmer-Rachamimov AO, Chiocca EA: Pattern of self-organization in tumour systems: complex growth dynamics in a novel brain tumour spheroid model. Cell Proliferation 200 I, 34(2): I I5-I34.

5. Sander LM, Deisboeck TS: Growth patterns of microscopic brain tumors. Physical Review E 2002, 66(5):051901.

6. Delsanto PP, Guiot C, Degiorgis PG, Condat CA, Mansury Y, Deisboeck TS: Growth model for multicellular tumor spheroids. Applied Physics Letters 2004, 85( I 8):4425-4227.

7. Mojsilovic ARB, Gomez J, Deisboeck TS: Analysis, reconstruction and visualization of malignant brain tumors: a case study in data synthesis. Analyt Quant Cytol Histol 2002, 24: I 25-133.

8. Aguayo JB, Blackband SJ, Schoeniger J, Mattingly MA, Hintermann M: Nuclear magnetic resonance imaging of a single cell. Nature 1986, 322(6075): 190-191.

9. Cho ZH, Ahn CB, Juh SC, Lee HK, Jacobs RE, Lee $S, Y i J H$, Jo JM: Nuclear magnetic resonance microscopy with 4-um resolution: Theoretical study and experimental results. Medical Physics 1988, I 5(6):824.

10. Johnson GAB, Thompson M, Gewalt SL, Hayes CE: Nuclear magnetic resonance imaging at microscopic resolution. Journal of Magnetic Resonance 1986, 68(I): I29-I37.

II. Shuning Huang DV, Weitz David A, Gangping Dai , Rosen Bruce R, Deisboeck Thomas S: Magnetic Resonance Microscopy of a Glioma Spheriod in a Collagen I Matrix. ISMRM I4th SCIENTIFIC MEETING \& EXHIBITION: 6-I2 May 2006; Seattle, Washington, USA 2006.
12. Nishikawa R, Ji X, Harmon RC, Lazar CS, Gill GN, Cavenee WK, Huang HS: A Mutant Epidermal Growth Factor Receptor Common in Human Glioma Confers Enhanced Tumorigenicity. PNAS 1994, 9 I(I6):7727-773I.

13. Nagane M, Coufal F, Lin H, Bogler O, Cavenee WK, Huang HJS: A Common Mutant Epidermal Growth Factor Receptor Confers Enhanced Tumorigenicity on Human Glioblastoma Cells by Increasing Proliferation and Reducing Apoptosis. Cancer Res 1996, 56(2I):5079-5086.

14. Kelm NET Jens M, Brown Catherine J, Fussenegger Martin, Nielsen Lars K: Method for generation of homogeneous multicellular tumor spheroids applicable to a wide variety of cell types. Biotechnology and Bioengineering 2003, 83(2): I73- 180.

15. Revel D, Brasch RC, Paajanen H, Rosenau W, Grodd W, Engelstad B, Fox P, Winkelhake J: Gd-DTPA contrast enhancement and tissue differentiation in MR imaging of experimental breast carcinoma. Radiology 1986, I 58(2):319-323.

16. Chen G, Jespersen SN, Pedersen M, Pang Q, Horsman MR, StodkildeJorgensen $\mathrm{H}$ : Intravenous administration of Gd-DTPA prior to DWI does not affect the apparent diffusion constant. Magnetic Resonance Imaging 2005, 23(5):685-689.

17. Abramoff MD, Magelhaes PJ, Ram SJ: Image Processing with ImageJ. Biophotonics International 2004, I I (7):36-42.

18. 3D Slicer [http://www.slicer.org/]

19. Giese A, Loo MA, Tran N, Haskett D, Coons SW, ME B: Dichotomy of astrocytoma migration and proliferation. Int J Cancer 1996 , 67(2):275-282

20. Athale CA, Deisboeck TS: The effects of EGF-receptor density on multiscale tumor growth patterns. Journal of Theoretical Biology 2006, 238(4):77I-779.

2I. Zhang L, Athale CA, Deisboeck TS: Development of a threedimensional multiscale agent-based tumor model: Simulating gene-protein interaction profiles, cell phenotypes and multicellular patterns in brain cancer. Journal of Theoretical Biology 2007, 244(I):96-107.

22. Maria Teresa Santini GR, Antonella Ferrante, Paola Indovina, Gianfranco Donelli, Pietro Luigi Indovina: A 50 Hz sinusoidal magnetic field does not damage MG-63 three-dimensional tumor spheroids but induces changes in their invasive properties. Bioelectromagnetics 2006, 27(2): |32-141.

23. Short W, Goodwill L, Taylor C: Alteration of human tumor cell adhesion by high-strength static magnetic fields. Investigative Radiology 1992, 27:836-840

24. Raylman ACC Raymond R, Wahl Richard L: Exposure to strong static magnetic field slows the growth of human cancer cells in vitro. Bioelectromagnetics 1996, I 7(5):358-363.

25. Brandl MTJ, Kotitschke K, Goldbrunner R, Kerkau S, Haase A: Quantitative NMR microscopy of multicellular tumor spheroids and confrontation cultures. Magnetic Resonance in Medicine 1995 , 34(4):596-603.

26. Helmchen F, Denk W: Deep tissue two-photon microscopy. Nat Meth 2005, 2( I 2):932-940.

27. McConnell G: Improving the penetration depth in multiphoton excitation laser scanning microscopy. Journal of Biomedical Optics 2006, I I(5):054020.

28. Raub CB, Suresh V, Krasieva T, Lyubovitsky J, Mih JD, Putnam AJ, Tromberg BJ, George SC: Noninvasive Assessment of Collagen Gel Microstructure and Mechanics Using Multiphoton Microscopy. Biophys J 2006. biophysj. I06.097998.

29. Nygaard SJT, Tysnes O-B: Quantification of glioma cell invasion by confocal laser scanning microscopy in an in vitro co-culture system. Cancer Letters 1996, I 05(I):45-49.

30. The Center for the Development of a Virtual Tumor, CViT [http://www.cvit.org]

\section{Pre-publication history}

The pre-publication history for this paper can be accessed here:

http://www.biomedcentral.com/1471-2342/8/3/prepub 